\title{
3-Level Degenerative Spondylolisthesis with Spinal Banal Stenosis
}

\author{
Moo Ing How', Tan See Wei', Kasat Niraj', Thng Leong Keng' \\ From the Department of Orthopaedics Surgery, Changi General Hospital, Singapore' and \\ University Malaya Medical Centre, Malaysia².
}

\section{Abstract:}

Introduction: Lumbar degenerative spondylolisthesis is a major cause of impaired quality of life and diminished functional capacity in the elderly. Degenerative spondylolisthesis often involves only one or two level and tend to present with one or two level spinal canal stenosis. Case Report: The authors describe an unusual case of degenerative spondylolisthesis involving 3 levels of the lumbar spine from L2 to L5. The patient was a 58 year old woman who suffered chronic back pain and neurogenic claudication. Plain radiography revealed grade I degenerative spondylolisthesis at L2-L3, L3-L4 and L4-L5. Elevated pedicle-facet joint angles and W-type facet joints at the lumbar spine was observed. Magnetic resonance imaging showed L2-S1 spinal cord compression at the lumbar spine. Patient underwent L2-S1 decompression laminectomy and posterior lateral fusion of L2-S1 with posterior instrumentation and bone grafting. Symptoms improved significantly at 4 months follow-up. Conclusion: Thorough evaluation for multilevel segmental involvement in degenerative spondylolisthesis is important because of the frequency of severe symptomatic spinal stenosis or foraminal encroachment. Good surgical outcome can be expected from decompression and stabilization. The pathogenesis of multi-level lumbar degenerative spondylolisthesis can be complex and heterogeneous.

Key words: Spondylolisthesis, Spinal stenosis, Lumbar Vertebrae, Spinal cord compression, Lumbosacral Region.

\section{Introduction}

Lumbar degenerative spondylolisthesis is a result of degenerative disorder of the intervertebral motion segment. It is a major cause of severe spinal canal stenosis and is often related to low back pain and leg pain. It often results in impaired quality of life and diminished functional capacity in the elderly. Degenerative spondylolisthesis often involved only one level $(62 \%)$ and tend to present with severe localized stenosis at L4-L5 level [1]. However, it is well known that degenerative spondylolisthesis occurs in multiple levels of the lumbar spine and the frequency of multilevel spondylolisthesis is reported to be 5-11\% [2]. Multilevel segmental involvement is considered to be important for cauda equina dysfunction and for evaluation of decompression levels. To the best of our knowledge, there has been no published report of 3-segments degenerative spondylolisthesis with severe L2-S1 spinal stenosis. This article is the first report on an unusual case of multilevel degenerative spondylolisthesis of

Corresponding Author: Dr. Ing How Moo

Email: zell53@hotmail.com

Received: June 7,2014 | Accepted: June 24, 2014 | Published Online: July 20, 2014

This is an Open Access article distributed under the terms of the Creative Commons Attribution License (creativecommons.org/licenses/by/3.0)

Conflict of interest: None declared | Source of funding: Nil | DOl: http://dx.doi.org/10.17659/01.2014.0065 
the lumbar spine in a patient with back pain and neurogenic claudication.

\section{Case Report}

This 58 year old female presented with 4 year history of low back pain that progressively worsen over the past 1 year and was associated with bilateral buttock, lateral thigh and calf pain along L4 and L5 dermatome. Pain was increased upon walking and relieved by rest. Claudication distance measured 5-10 meters. Physical examination revealed tenderness over the lumbar spine. Lumbar flexion and extension were limited and painful. There was weakness of the bilateral extensor hallucis longus
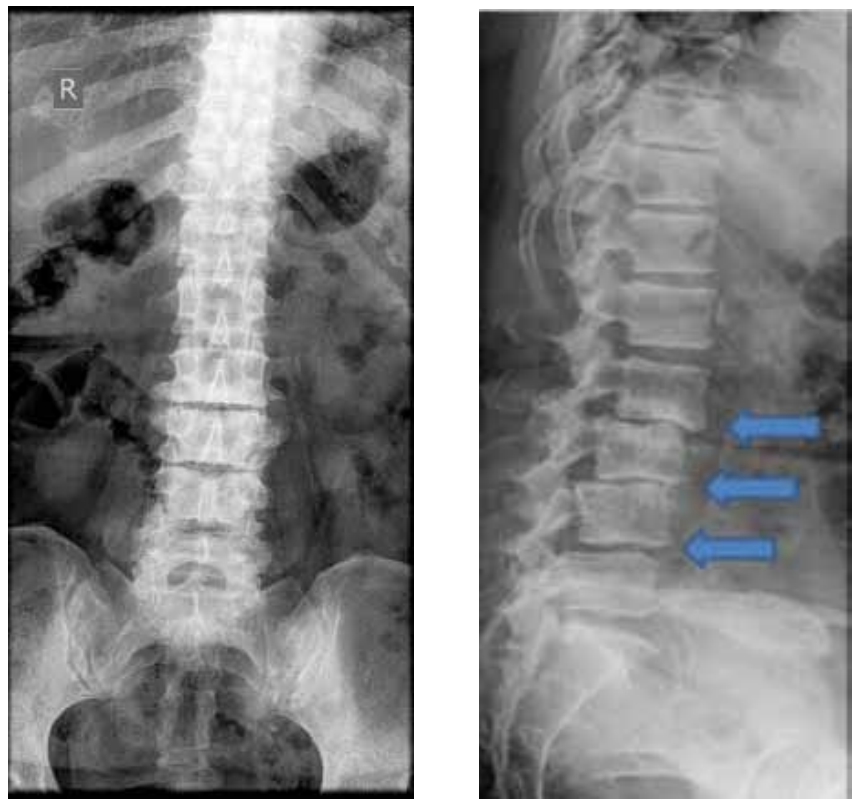

Fig.1: Plain radiographs of the lumbar vertebrae, anteroposterior (Left) and lateral neutral (Right) view, showing grade I degenerative spondylolisthesis at L2-L3, L3-L4 and L4-L5 associated with disc space narrowing. The pedicle-facet angles were $127^{\circ}$ at $L 2,126^{\circ}$ at $L 3,123^{\circ}$ at $L 4$, and $124^{\circ}$ at L5. The amount of slip over L2-L3, L3-L4 and L4-L5 were $9 \mathrm{~mm}, 7$ $\mathrm{mm}$ and $6 \mathrm{~mm}$, respectively. (i.e. muscle strength grading of $4 / 5$ ) and sensation at L4 and L5 dermatome was reduced. Plain radiography of the lumbar vertebrae revealed grade I degenerative spondylolisthesis at L2-L3, L3-L4 and L4-L5 [Fig.1,2]. Radiological studies showed elevated pedicle-facet angle and W- type of facet joints from L2-L5. Magnetic resonance imaging revealed showed severe multilevel spinal canal stenosis from L2-S1 [Fig.3].

L2-S1 decompression laminectomy and posterior lateral fusion of L2-S1 with posterior instrumentation and bone graft was performed to decompress the spinal cord and stabilize the lumbar spine. Intraoperatively, patient was found to have severe degenerative stenosis from L2 to S1 level. Patient underwent an uneventful postoperative recovery [Fig.4]. At a recent follow-up, 4 months after the surgery, the symptoms of the patient were significantly improved. Patient was ambulating without aid and did not complain of any leg symptoms.

\section{Discussion}

The earliest description of degenerative
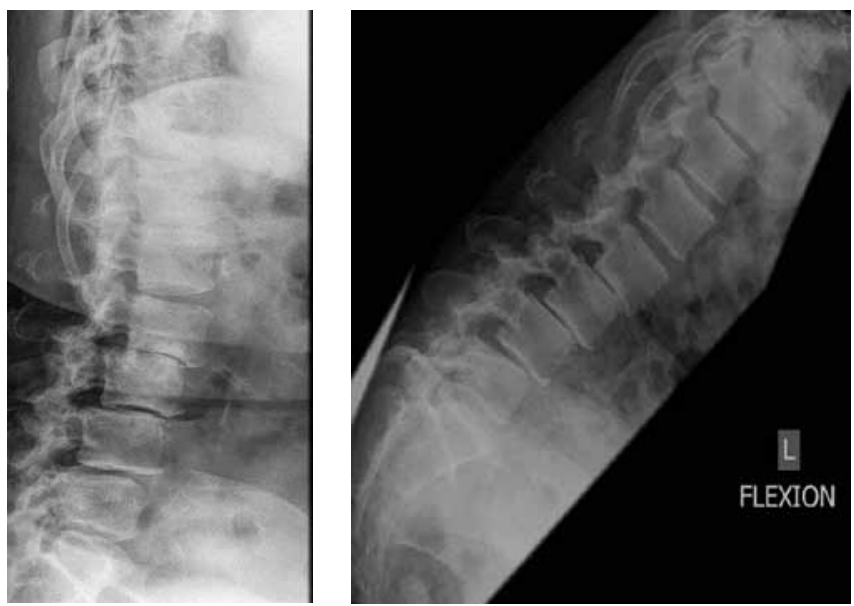

Fig.2: Flexion (Left) and extension (Right) view of the lumbar vertebrae showed a stable lumbar spine. 

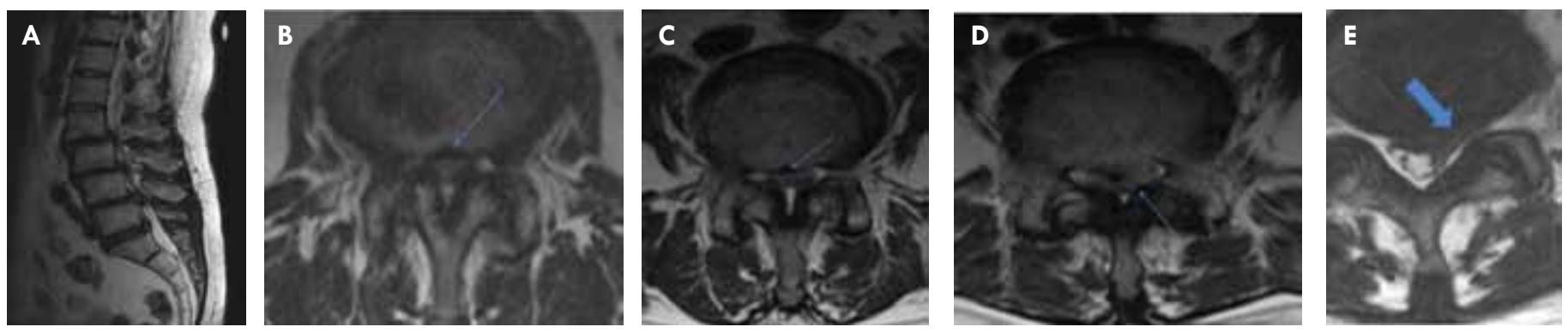

Fig.3: T2 weighted images of the Lumbar spine- sagittal (A) and axial [L2-L3 (B), L3-L4 (C), L4-L5 (D), L5S1 (E)] demonstrating L2 to L5 diffuse disc bulge, bilateral facet joint degeneration and ligamentum flavum hypertrophy resulting in the severe central canal, subarticular zones and exit foramina stenosis. At L5/S1 level, central and left paracentral disc protrusion has resulted in moderate narrowing of the central canal and bilateral subarticular zones (i.e. the left subarticular zone is narrowed to a greater degree).

spondylolisthesis dates back to the $19^{\text {th }}$ century. Junghanns, in 1930, defined lumbar vertebral slippage without a pars interarticularis defect as "pseudospondylolisthesis". The modern concept, in use today, was later described by Newman in 1955 [3]. This occurs mostly at L4-L5 in adults older than 40 years. There have been many postulated causative factors of anterolisthesis. However, to our knowledge, there have been no reports as to whether multilevel slip is caused by factors similar to those observed in single-level slip. A radiological analysis of our case has suggested that the pathogenesis of multilevel lumbar degenerative spondylolisthesis can be complex and heterogeneous.

Individuals with degenerative spondylolisthesis had a greater mean facet joint angle, as demonstrated in our case (averaging $125^{\circ}$ which is significantly higher than those reported in the literature). High pedicle-facet angle reduces the ability of the facet joints to resist the forward displacement force and can strain the intervertebral disc which eventually underwent degeneration. When the disc degenerates, the axis of flexion and extension shift posteriorly to the facet joint. Such alteration may contribute to the development of facet joint osteoarthritis which does not retain anterior shear forces and eventually results in spondylolisthesis
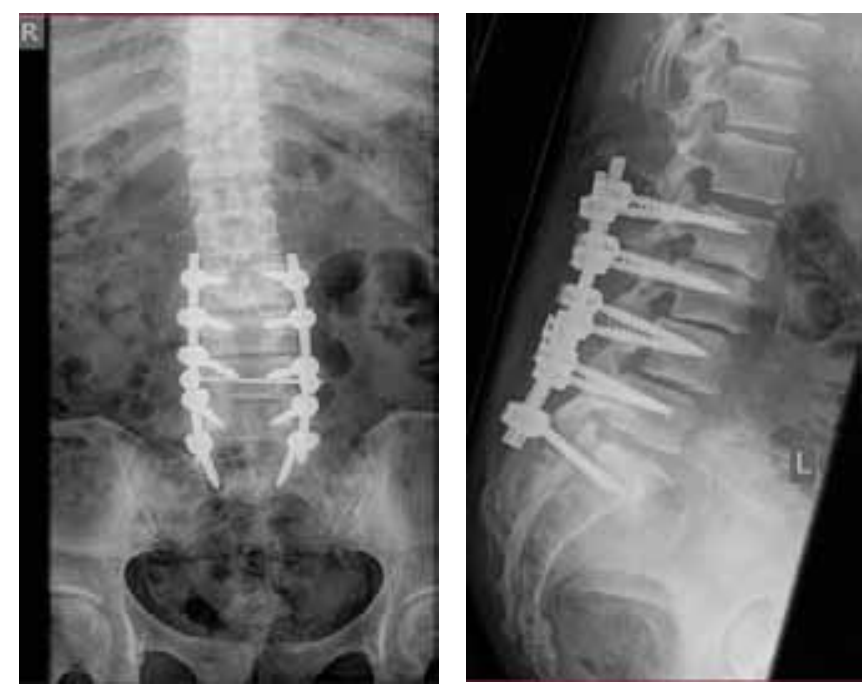

Fig.4: Postoperative anteroposterior (Left) and lateral (Right) $x$-rays showing L2-S1 decompression laminectomy and posterior instrumentation at 4 months follow-up.

[4]. Secondary changes can be observed in the rest of the spine. There may be increased lumbar curvature, facet joints osteoarthritis, pelvic rotation and ultimately generalized lumbar arthritis. The degree of slip is usually $4-5 \mathrm{~mm}$ or less than $25 \%$ according to the Meyerding classification [2]. However, since the neural arch is intact, even a small degree of slip can cause neural compression within 
spinal canal. The associated hypertrophy of the ligamentum flavum or protrusion or bulging of the intervertebral disc further worsened the situation. All these pathological changes explained the sensory or motor disturbances (i.e. low back pain, leg pain, neurogenic claudication) in our index patient.

Furthermore, the orientation of the facet joints appears to be a risk factor. The body-weight transmits a forward and downward vector force to the L5 level, but this force is resisted by the soft tissues and the posterior articulations. When the facet joints are in the sagittal plane there is relatively less bony resistance to anterior shear force compare to joints that are in oblique or coronal orientation [5]. Our case showed a sagittal facet joint orientation at the level L3-L4 and L4-L5, 38.5 and $26.5^{\circ}$ respectively. Contrarily to the findings of other authors, our patient did not reveal sagittal facet joint orientation at L2-L3 and L5-S1, 48 and $45^{\circ}$ respectively. It remains a debate whether sagittal alignment is a pre-existing morphological feature or a result of secondary remodelling of the joint orientation [6]. Nonetheless, a W-shaped facet joint is reported to have a close relationship with the sagittal orientation of the facet joint.

Articular tropism at L5 may be very important in predisposing to spondylolisthesis. This asymmetry is not conducive for proper biomechanics or stability. In addition, sacralisation of L5 is 4 times more common in degenerative spondylolisthesis population. This suggests that a relatively immobile L5/S 1 articulation tends to predispose $L 4 / 5$ to added stress [5]. However, such anatomic anomalies are not seen in our case.

Roussouly et al. described 4 typical subtypes of sagittal orientation of the lumbar spine and pelvis. Type IV of this classification is characterized by a high sacral slope and a high pelvic incidence with associated significant lumbar lordosis. Such spinopelvic configuration has a relatively vertical
S1 endplate in the sagittal plane and might predispose to anterior displacement of the lumbar vertebrae above [6]. However, such predisposing anatomic feature is not observed in our case.

Increased body mass index has also been found to be a predisposing factor for degenerative spondylolisthesis due to increased axial load on the vertebrae. Pregnancy, generalized joint laxity, and oophorectomy are thought to be predisposing factors of the condition and underlie its predominance in women (sex ratio 4 females to 1 male) [2].

\section{Conclusion}

Thorough evaluation for multilevel segmental involvement in degenerative spondylolisthesis is important because of the frequency of severe symptomatic spinal stenosis or foraminal encroachment. Good surgical outcome can be expected from decompression and stabilization of multi-level degenerative spondylolisthesis. The pathogenesis of multi-level lumbar degenerative spondylolisthesis can be complex and heterogeneous.

\section{References}

1. Park DK, An HS, Lurie JD, Zhao W, Tosteson A, Tosteson TD, et al. Does multilevel lumbar stenosis lead to poorer outcomes: a subanalysis of the Spine Patient Outcomes Research Trial (SPORT) lumbar stenosis study. Spine (Phila Pa 1976) $2010 ; 35(4): 439-446$.

2. Iguchi T, Wakami T, Kurihara A, Kasahara K, Yoshiya S, Nishida K. Lumbar multilevel degenerative spondylolisthesis: radiological evaluation and factors related to anterolisthesis and retrolisthesis. J Spinal Disord Tech 2002; 1 5(2):93-99.

3. Newman PH. Spondylolisthesis, its cause and effect. Ann R Coll Surg Engl 1955;16(5):305323. 
4. Macnab I. Spondylolisthesis with an intact neural arch; the so-called pseudo-spondylolisthesis. J Bone Joint Surg Br 1950;32-B(3):325-333.

5. Rothman SL, Glenn WV Jr, Kerber CW. Multiplanar CT in the evaluation of degenerative spondylolisthesis. A review of 150 cases.
Comput Radiol 1985;9(4):223-232.

6. Schuller S, Charles YP, Steib JP. Sagittal spinopelvic alignment and body mass index in patients with degenerative spondylolisthesis. Eur Spine J 2011 ;20(5):713-719. 